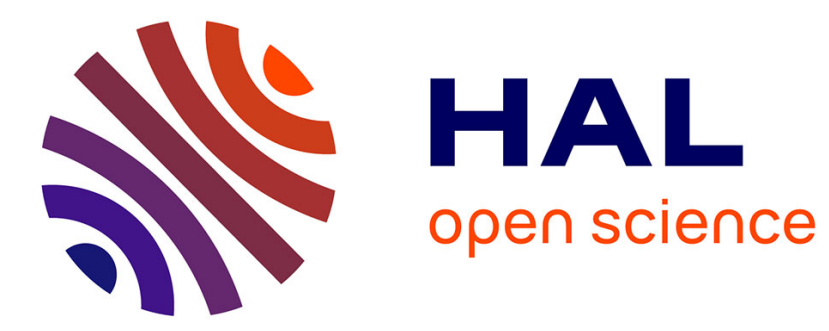

\title{
Practical Role of Racemization Rates in Deracemization Kinetics and Process Productivities
}

\author{
Ryusei Oketani, Marine Hoquante, Clément Brandel, Pascal Cardinaël, \\ Gérard Coquerel
}

\section{- To cite this version:}

Ryusei Oketani, Marine Hoquante, Clément Brandel, Pascal Cardinaël, Gérard Coquerel. Practical Role of Racemization Rates in Deracemization Kinetics and Process Productivities. Crystal Growth \& Design, 2018, 18 (11), pp.6417-6420. hal-01950306

\section{HAL Id: hal-01950306 \\ https://hal-normandie-univ.archives-ouvertes.fr/hal-01950306}

Submitted on 3 Sep 2019

HAL is a multi-disciplinary open access archive for the deposit and dissemination of scientific research documents, whether they are published or not. The documents may come from teaching and research institutions in France or abroad, or from public or private research centers.
L'archive ouverte pluridisciplinaire HAL, est destinée au dépôt et à la diffusion de documents scientifiques de niveau recherche, publiés ou non, émanant des établissements d'enseignement et de recherche français ou étrangers, des laboratoires publics ou privés. 


\title{
Practical role of racemization rates in deracemization kinetics and process productivities
}

\author{
Ryusei Oketani, Marine Hoquante, Clément Brandel, Pascal Cardinael, Gérard Coquerel* \\ Normandie Université, Laboratoire SMS-EA3233, Université de Rouen, F-76821, Mont Saint Aignan, France
}

Supporting Information Placeholder

\begin{abstract}
Herein, we report the chiral symmetry breaking of 2-methoxy-1-naphthamide atropisomers through temperature cycling without the use of any racemization reagent. The racemization rate $\left(k_{1}\right)$ controls the deracemization process when the cooling of the slurry is slow enough to keep the system close to equilibrium. The productivity appears proportional to the racemization rate $\left(k_{1}\right)$ multiplied by the solubility.
\end{abstract}

Chirality has attracted research interest for many years. Among many of its fascinating facets, chiral resolution, i.e., the process by which two mirror-image molecules are separated from their mixture, is of paramount importance in chemistry. ${ }^{1-3}$ In the context of the pharmaceutical industry, this has led to the stringent requirement that any new chiral pharmaceutical ingredient be developed in its active enantiopure form. ${ }^{4,5}$ Hence, resolution procedures are important; optimization facilitated by a fundamental understanding of the resolution process is key to the successful application of these techniques in industry.

Among the different chiral-resolution techniques available to the engineer, ${ }^{6-9}$ deracemization is an attractive process because its theoretical yield is $100 \%$, whereas the yield from other methods cannot exceed $50 \%$. Although many techniques, for example grinding, ${ }^{10,11}$ ultrasound, ${ }^{12}$ homogenization, ${ }^{13}$ temperature cycling, ${ }^{14,15}$ and microwave ${ }^{16}$ have been applied to deracemization processes, temperature cycling is considered to be the most favourable technique from an industrial perspective because of its simplicity and applicability to a wide range of compounds. Also, in the context of the universal origin of chirality, temperature cycling is found in the natural day/night cycle, which might induce (or promote) deracemization in a natural system. Hence, clarification of the key parameter(s) that control(s) deracemization processes through temperature cycling is urgently required, not only from an industrial viewpoint, but also from the perspective of gaining a universal understanding of symmetry breaking.

While many molecules have been deracemized, several researchers have attempted to experimentally identify the key parameters that control these processes. ${ }^{17-30}$ The results of these studies suggest that racemization and crystal-growth/dissolution rates are essential factors that govern the efficiency of a deracemization procedure. This observation is in good agreement with that of Iggland and Mazzotti, who focused on computational simulations of the isothermal Viedma ripening process, and showed that the ratio of these rates is the key criterion that controls the deracemization rate. ${ }^{26}$ However, in the case of deracemization by temperature cycling, dissolution/growth rates are mainly controlled by the temperature profiles.

Consequently, when dissolution and crystal growth are performed smoothly, racemization rates should be, in principle, the key parameter in deracemization kinetics. In addition, if the racemization rate alone is a good proxy for estimating deracemization, it will provide a high level of practical convenience.

Despite this background, the quantitative role of racemization rate on deracemization kinetics has not been considered experimentally yet because of the difficulties associated with experimental systems. Indeed, most of the published deracemization studies focus on chiral compounds containing stereogenic centres that follow complicated deracemization kinetics because an additional component (e.g. a base) is added to the system, thereby adding an additional level of complexity to the problem. ${ }^{31}$ The deracemization of atropisomers elegantly circumvents this issue. ${ }^{11}$ Indeed, atropisomerism arises from axial chirality and is due to restricted rotation about a single bond; accordingly, its racemization is a unimolecular process that should follow first-order reaction kinetics characterized by a single parameter.

In this context, we prepared naphthamide $\mathbf{1}$ as a compound that racemizes in a simple manner and forms in conglomerate system. Indeed, compound 1 exhibits frozen atropisomerism in its solidstate, whereas it spontaneously racemizes in solution. Naphthamide 1 has previously been reported by Sakamoto et al., ${ }^{32}$ and our sample was synthesized using a similar route (Scheme 1), the details of which can be found in the Supporting Information. Large single crystals $(5-30 \mathrm{mg})$ for racemization-rate experiments were also prepared by the slow evaporation of a saturated 1:1 (v/v) $\mathrm{CHCl}_{3}$ :heptane solution.

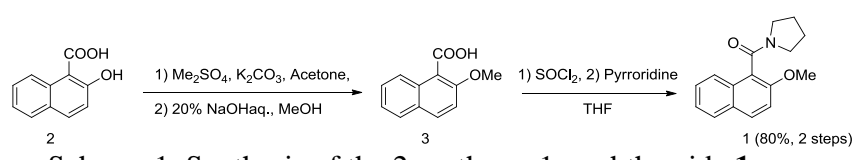

Scheme 1. Synthesis of the 2-methoxy-1-naphthamide $\mathbf{1}$.

Sakamoto et al. reported the racemization rate constant $\left(k_{1}\right)$ for $\mathbf{1}$ in methanol (MeOH), tetrahydrofuran (THF), and a 1:1 mixture of $\mathrm{MeOH}$ and THF. ${ }^{32}$ Here, we measured $k_{1}$ in various solvents including alcohols, ketones, nitriles, and ethers. A large single crystal (consisting of only one chiral form) was dissolved in a solvent and the time dependence of the optical rotation at $365 \mathrm{~nm}$ was recorded over a few hours using a polarimeter. Hence, the slope of $\ln \left[\alpha_{t} / \alpha_{0}\right]$ 
as a function of time was used to determine the first-order racemization rate constant $k_{1}$. The racemization rate was evaluated in terms of racemization rate constant $k_{1}$.

The solubilities and racemization rate constants $\left(k_{1}\right)$ of $\mathbf{1}$ in the solvents considered are summarized in Table S1 in the Supporting Information. Generally, $k_{1}$ was higher in the less-polar solvents. For example, $k_{1}$ increased linearly with increasing solvent chain length in the nitrile solvents. Although the racemization rate constants were highest in the ether solvents, solubility was relatively low. The racemization rate constants were much lower in the alcohol series than in other types of solvent. These observations are ascribable to hydrogen bonding between the carbonyl oxygen of $\mathbf{1}$ and the solvent, which reduces the rate of racemization. Although Sakamoto et al. reported that $\mathbf{1}$ retains its chirality in $\mathrm{MeOH}$ through hydrogen-bonding interactions, ${ }^{32}$ our systematic study reveals that 1 racemizes at a rate depending on the solvent polarity; this trend is particularly conspicuous within the same solvent series.

Deracemization experiments involving 1 were carried out in $t \mathrm{BuOH}, \mathrm{MTBE}, \mathrm{MIBK}$, and $i \mathrm{PrCN}$ by temperature cycling of the saturated solution in equilibrium with the crystals, as described in the following procedure. First, the atropisomer was dissolved in the solvent and the solution was filtered through a membrane filter. The filtered solution was maintained at $20^{\circ} \mathrm{C}$ until crystallization spontaneously occurred. The first crystals are usually slightly $(<3 \%)$ randomly enriched with one enantiomer. Temperature cycling was then performed between 20 and $25^{\circ} \mathrm{C}$ for $5-7 \mathrm{~d}$. The enantiomeric excess of the solid was monitored by optical rotation every $24 \mathrm{~h}$. Since the cooling from 25 to $20{ }^{\circ} \mathrm{C}$ corresponds to the crystalgrowth step, it was conducted more slowly than the other steps in order to prevent an uncontrolled nucleation of the counter enantiomer. We showed in this study that the racemization of $\mathbf{1}$ occurs very slowly in cold methanol $(\mathrm{MeOH})$; therefore, these conditions were selected to evaluate optical rotation and to mitigate the racemization of 1 .

It should be noted that $20 \%$ of the crystal mass was deliberately dissolved during heating in order to directly compare the deracemization rates in different solvents (Figure 1). The solution equilibria after the heating and cooling steps were confirmed in the same manner used in gravimetric-solubility experiments; i.e., the times for dissolution and crystal growth were sufficient as not to be the rate-limiting steps in the experiment.

Compound 1 was efficiently deracemized within a few days with final enantiomeric excesses (ees) greater than $90 \%$ in $t \mathrm{BuOH}$,

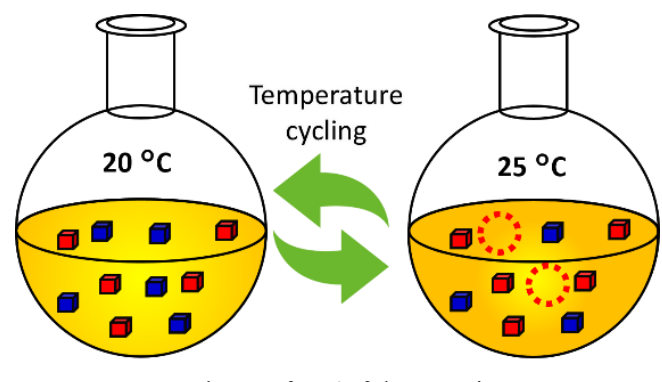

Dissolution of $20 \%$ of the crystals

Figure 1. Schematic illustration of the deracemization experiment.

MTBE, MIBK, and $i \operatorname{PrCN}$, through temperature cycling, with or without an initial chiral bias (Figure 2). The crystal form obtained by deracemization was the same as that reported earlier. ${ }^{32}$

The evolution of enantiomeric excess was sigmoidal in nature. This behaviour is often observed in solid-state deracemizations. The dominant enantiomer was determined by the initial chiral bias, except in the $i \mathrm{PrCN}$ system. Indeed, in the latter case, when the
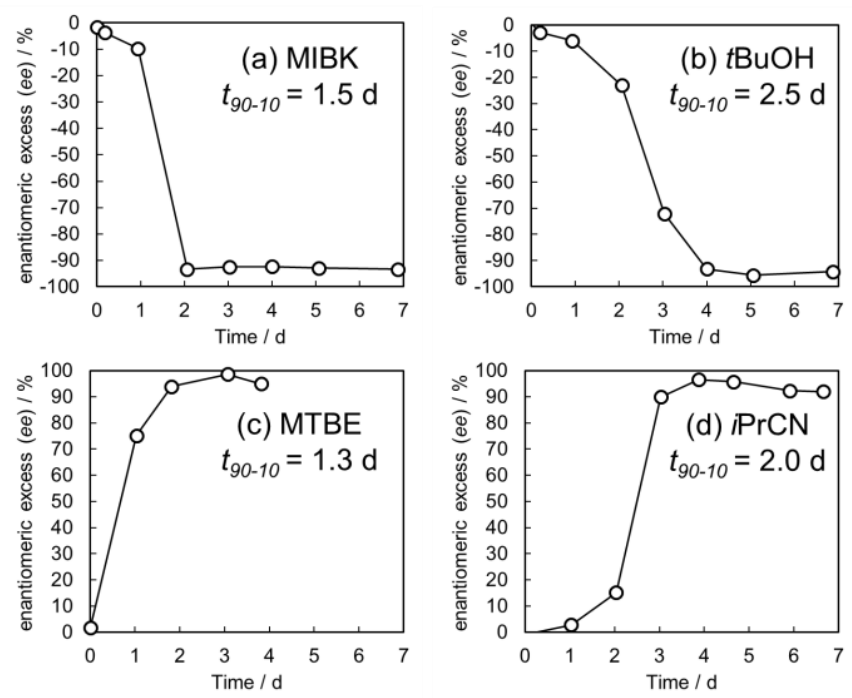

Figure 2. Enantiomeric excess (ee) values of the collected crystals of 1 as functions of time during deracemization experiments, and the corresponding t90-10 values in (a) MIBK, (b) $t \mathrm{BuOH}$, (c) $\mathrm{MTBE}$, and (d) $i \mathrm{PrCN}$. Each point includes uncertainty $(<$ $\pm 1.5 \%$ ) due to the polarimetery technique.

process was slightly (-)-enriched, deracemization occurred with the formation of the (opposite) (+)-isomer. Although we observed this phenomenon on two occasions, we are currently unable to provide an explanation for this observation.

To compare the deracemization rates under different conditions, Iggland and Mazzotti used the time required for the racemic mixture $(0 \% e e)$ to reach $90 \% e e$ in order to evaluate isothermal Viedma ripening in their computational work. ${ }^{26}$ Here, we define the time required to reach $90 \%$ ee from a slightly enriched state $(10 \%$ $e e$ ), to be $t 90-10$, which excludes unreproducible behaviour often observed at the start of the deracemization process. For example, chirality in the solid state can deviate to one enantiomer and then heads in the opposite direction. The $t_{90-10}$ values reported in Figure 2 are the averages of experiments conducted in triplicate.

According to Iggland and Mazzotti, deracemization kinetics is correlated theoretically to the ratio between the racemization rate and the crystal growth/dissolution rate. ${ }^{26}$ However, as mentioned earlier, the cooling/heating steps were sufficiently slow, see also supporting information, to ensure that the suspension remains close to equilibrium; consequently these steps are not rate limiting and can be excluded when considering the overall deracemization rate in the experiment. Thus, herein, we considered the influence of the racemization rate on the global deracemization rate.

Figure 3a displays a plot of the natural logarithm of $k_{1}$ as a function of $t_{90-10}$ using the experimental data collected herein for $\mathbf{1}$ in the four different solvents. It reveals that the deracemization rate can be estimated by simply measuring the racemization rate in the case of temperature cycling. Contextualising to real phenomena, these parameters can be intuitively interpreted as follows. Slow racemization in solution retains chirality for long periods of time and crystallization occurs with the same chirality before dissolution; therefore, deracemization proceeds at a sluggish pace. By contrast, the solution remains racemic during fast racemization, even during crystallization, which contributes to deracemization.

This behaviour qualitatively rationalizes other results obtained experimentally for deracemization; for example, Breveglieri et al. recently reported the deracemization of $\mathrm{N}$-(2-methylbenzylidene)phenylglycine amide (NMPA) with DBU through temperature cycling, and concluded that a high operating temperature leads to fast deracemization. ${ }^{15}$ Racemization of NMPA with DBU can be 

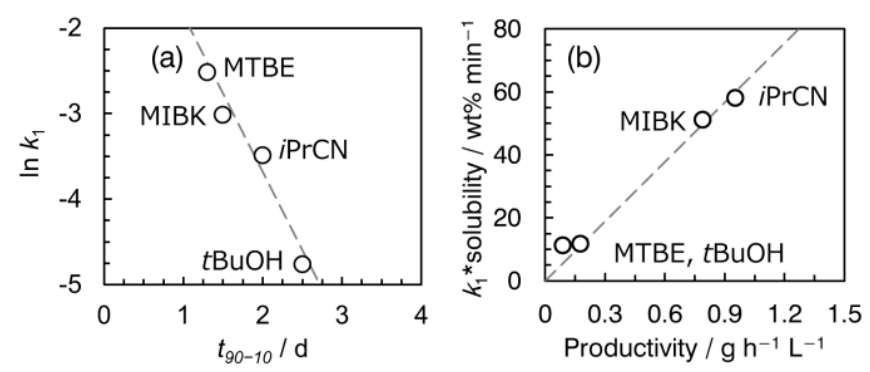

Figure 3. (a) Time required to achieve an ee of $90 \%($ t90-10) as a function of natural logarithm of the racemization rate $\left(k_{1}\right)$. (b) The product of $k_{1}$ and solubility as a function of deracemization productivity.

considered to be a catalytic reaction; consequently, the racemization rate is higher at higher operating temperatures. The faster deracemization kinetics at higher temperature for NMPA with DBU is, according to our interpretation, mostly due to the high racemization rate of NMPA.

Moreover, we found that the deracemization productivity, which is defined by how much of the desired enantiomer is produced per unit time and volume of suspension, correlates linearly with the product of $k_{1}$ and solubility, as shown in Figure $3 \mathrm{~b}$. In terms of the global outcome of the process, $k_{1}$ controls the deracemization time, and solubility determines the scale of the symmetry breaking; hence, their product provides the productivity. For example, MTBE yields the fastest deracemization, but it is the worst medium in terms of productivity because of the poor solubility. This contrast illustrates that the order of the productivity differs from that of the deracemization rate. Thus, to achieve practically feasible applications of the process, it is very important to consider the balance between $k_{1}$ and the solubility.

In summary, we reported the first deracemization study of a single-component atropisomer through temperature cycling without the use of any racemization reagent. We also measured the racemization rates of the compound in different solvents to understand its influence on the overall deracemization rate. Our study suggests that the racemization rate $\left(k_{1}\right)$ is the key parameter that determines the deracemization kinetics during temperature cycling. When considering once again the report of Breveglieri et al., ${ }^{15}$ the faster deracemization kinetics at higher temperature for NMPA is probably also due to the higher solubility of NMPA.

Nevertheless, when the productivity of the global process is of interest, the product of racemization rate by solubility becomes the key parameter to consider. In this context, further process understanding can also be gained by studying the influence of impurities and cooling rate on the process kinetics, in addition to the starting particle-size distribution.

\section{ASSOCIATED CONTENT}

\section{Supporting Information}

The Supporting Information is available free of charge on the ACS Publications website.

Synthesis of 2-methoxy-1-naphthamide, and (2-methoxynaphthalen-1-yl)(pyrrolidin-1-yl)methanone, racemisation kinetics, crystal growth rate, deracemisation experiments (PDF), Movies monitoring crystal growth in MIBK (AVI).

\section{AUTHOR INFORMATION}

\section{Corresponding Author}

E-mail: gerard.coquerel@univ-rouen.fr

ORCID

Ryusei Oketani: 0000-0001-7860-2456

Gérard Coquerel: 0000-0001-8977-8676

Notes

The authors declare no competing financial interests.

\section{ACKNOWLEDGMENT}

This research received funding as part of a CORE project (October 2016-September 2020) from the Horizon 2020 Research and Innovation Programme of the European Union under Marie Sklodowska-Curie grant agreement No. 722456 CORE ITN.

\section{REFERENCES}

(1) Ribó, J. M.; Hochberg, D.; Crusats, J.; El-Hachemi, Z.; Moyano, A. Spontaneous Mirror Symmetry Breaking and Origin of Biological Homochirality. J. R. Soc. Interface 2017, 14, 20170699.

(2) Chubb, J. R. Symmetry Breaking in Development and Stochastic Gene Expression. Wiley Interdiscip. Rev. Dev. Biol. 2017, 6, e284

(3) Pillai, A. S.; Jirsa, V. K. Symmetry Breaking in Space-Time Hierarchies Shapes Brain Dynamics and Behavior. Neuron 2017, 94, 1010-1026.

(4) Francotte, E.; Lindner, W. Chirality in Drug Research; Francotte, E., Lindner, W., Eds.; Methods and Principles in Medicinal Chemistry; Wiley-VCH Verlag GmbH \& Co. KGaA: Weinheim, FRG, 2006.

(5) Nguyen, L. A.; He, H.; Pham-Huy, C. Chiral Drugs: An Overview. Int. J. Biomed. Sci. 2006, 2, 85-100.

(6) Guy, L.; Crassous, J.; Andraud, C. CRC Handbook of Optical Resolutions via Diastereomeric Salt Formation, Edited by David Kozma. Chirality 2002, 14, 820-820.

(7) Coquerel, G. Preferential Crystallization. Top. Curr. Chem. 2006, 269, 1-51.

(8) Anderson, N. G. Developing Processes for CrystallizationInduced Asymmetric Transformation. Org. Process Res. Dev. 2005, 9, 800-813.

(9) Breuer, M.; Ditrich, K.; Habicher, T.; Hauer, B.; Keßeler, M.; Stürmer, R.; Zelinski, T. Industrial Methods for the Production of Optically Active Intermediates. Angew. Chem. Int. Ed. 2004, $43,788-824$.

(10) Noorduin, W. L.; Bode, A. A. C.; van der Meijden, M.; Meekes, H.; van Etteger, A. F.; van Enckevort, W. J. P.; Christianen, P. C. M.; Kaptein, B.; Kellogg, R. M.; Rasing, T.; et al. Complete Chiral Symmetry Breaking of an Amino Acid Derivative Directed by Circularly Polarized Light. Nat. Chem. 2009, 1, 729-732.

(11) Engwerda, A. H. J.; van Schayik, P.; Jagtenberg, H.; Meekes, H.; Rutjes, F. P. J. T.; Vlieg, E. Solid Phase Deracemization of an Atropisomer. Cryst. Growth Des. 2017, 17, 5583-5585. Rougeot, C.; Guillen, F.; Plaquevent, J. C.; Coquerel, G. Ultrasound-Enhanced Deracemization: Toward the Existence of Agonist Effects in the Interpretation of Spontaneous Symmetry Breaking. Cryst. Growth Des. 2015, 15, 2151-2155.

(13) Iggland, M.; Fernández-Ronco, M. P.; Senn, R.; Kluge, J.; Mazzotti, M. Complete Solid State Deracemization by High Pressure Homogenization. Chem. Eng. Sci. 2014, 111, 106-111. Suwannasang, K.; Flood, A. E.; Coquerel, G. A Novel Design Approach to Scale Up the Temperature Cycle Enhanced Deracemization Process: Coupled Mixed-Suspension Vessels. Cryst. Growth Des. 2016, 16, 6461-6467. 
(15) Breveglieri, F.; Maggioni, G. M.; Mazzotti, M. Deracemization of NMPA via Temperature Cycles. Cryst. Growth Des. 2018, 18, 1873-1881.

(16) Kacker, R.; Radoiu, M.; Kramer, H. J. M. Novel Design Integrating a Microwave Applicator into a Crystallizer for Rapid Temperature Cycling. A Direct Nucleation Control Study. Cryst. Growth Des. 2017, 17, 3766-3774.

(17) Fytopoulos, A. A.; Xiouras, C.; Kavousanakis, M. E.; Van Gerven, T.; Boudouvis, A. G.; Stefanidis, G. D. A Population Balance Model for Temperature Cycling-Enhanced Deracemization. Cryst. Growth Des. 2018, acs.cgd.8b00706.

(18) Noorduin, W. L.; Meekes, H.; Van Enckevort, W. J. P.; Millemaggi, A.; Leeman, M.; Kaptein, B.; Kellogg, R. M.; Vlieg, E. Complete Deracemization by Attrition-Enhanced Ostwald Ripening Elucidated. Angew. Chem. Int. Ed. 2008, 47, 6445-6447.

(19) Xiouras, C.; Ter Horst, J. H.; Van Gerven, T.; Stefanidis, G. D. Coupling Viedma Ripening with Racemic Crystal

Transformations: Mechanism of Deracemization. Cryst. Growth Des. 2017, 17, 4965-4976.

(20) Xiouras, C.; Fytopoulos, A. A.; Ter Horst, J. H.; Boudouvis, A. G.; Van Gerven, T.; Stefanidis, G. D. Particle Breakage Kinetics and Mechanisms in Attrition-Enhanced Deracemization. Cryst. Growth Des. 2018, 18, 3051-3061.

(21) Uwaha, M. A Model for Complete Chiral Crystallization. $J$. Phys. Soc. Japan 2004, 73, 2601-2603.

(22) Katsuno, H.; Uwaha, M. Effect of Nucleation on Chirality Conversion Induced by Random Fluctuation. J. Cryst. Growth 2014, 401, 59-62.

(23) Uwaha, M. Simple Models for Chirality Conversion of Crystals and Molecules by Grinding. J. Phys. Soc. Japan 2008, 77, 8-11.
(24) Uwaha, M. Steady Chirality Conversion by Grinding Crystals Supercritical and Subcritical Bifurcations. J. Cryst. Growth 2011, 318, 89-92.

(25) Uwaha, M.; Katsuno, H. Mechanism of Chirality Conversion by Grinding Crystals: Ostwald Ripening vs Crystallization of Chiral Clusters. J. Phys. Soc. Japan 2009, 78, $2-5$.

(26) Iggland, M.; Mazzotti, M. A Population Balance Model for Chiral Resolution via Viedma Ripening. Cryst. Growth Des. 2011, 11, 4611-4622.

(27) Iggland, M.; Müller, R.; Mazzotti, M. On the Effect of Initial Conditions in Viedma Ripening. Cryst. Growth Des. 2014, 14, 2488-2493.

(28) Hein, J. E.; Huynh Cao, B.; Viedma, C.; Kellogg, R. M.; Blackmond, D. G. Pasteur's Tweezers Revisited: On the Mechanism of Attrition-Enhanced Deracemization and Resolution of Chiral Conglomerate Solids. J. Am. Chem. Soc. 2012, 134, 12629-12636.

(29) Gherase, D.; Conroy, D.; Matar, O. K.; Blackmond, D. G. Experimental and Theoretical Study of the Emergence of Single Chirality in Attrition-Enhanced Deracemization. Cryst. Growth Des. 2014, 14, 928-937.

(30) Uchin, R.; Suwannasang, K.; Flood, A. E. Model of Temperature Cycle-Induced Deracemization via Differences in Crystal Growth Rate Dispersion. Chem. Eng. Technol. 2017, 40, $1252-1260$.

(31) Yuchun, X.; Huizhou, L.; Jiayong, C. Kinetics of Base Catalyzed Racemization of Ibuprofen Enantiomers. Int. J. Pharm. 2000, 196, 21-26.

(32) Sakamoto, M.; Unosawa, A.; Kobaru, S.; Saito, A.; Mino, T.; Fujita, T. Asymmetric Photocycloaddition in Solution of a Chiral Crystallized Naphthamide. Angew. Chem. Int. Ed. 2005, $44,5523-5526$. 
Authors are required to submit a graphic entry for the Table of Contents (TOC) that, in conjunction with the manuscript title, should give the reader a representative idea of one of the following: A key structure, reaction, equation, concept, or theorem, etc., that is discussed in the manuscript. Consult the journal's Instructions for Authors for TOC graphic specifications.

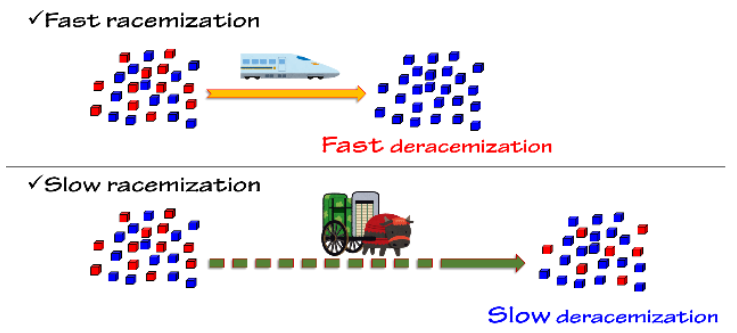

